\title{
Atmospheric Neutrino Oscillations at Super-Kamiokande
}

\author{
Roger Wendell, for the Super-Kamiokande Collaboration* \\ Institute for Cosmic Ray Research, The University of Tokyo \\ E-mail: rawasuketto.icrr.u-tokyo.ac.jp
}

\begin{abstract}
Spanning several orders of magnitude in both neutrino energy and path length, atmospheric neutrinos are a versatile probe of both standard and exotic mixing scenarios. Indeed, recent measurements of $\theta_{13}$ by reactor antineutrino experiments have opened up the possibility to observe the effect of the earth's matter on neutrino oscillations and to subsequently determine the neutrino mass hierarchy using atmospheric neutrinos. Further, the existence of a sterile neutrino outside of the standard three-neutrino oscillation paradigm would produce observable distortions in the atmospheric neutrino flux that can be used to probe hints from short-baseline oscillation experiments supporting an additional neutrino. Atmospheric neutrinos can similarly be used to explore possible deviations from Lorentz invariance and are particularly sensitive to violations of this symmetry that induce oscillation effects that scale with the neutrino energy and path length. The latest results from searches for each of these phenomena using Super-Kamiokande atmospheric neutrino are discussed.
\end{abstract}

The 34th International Cosmic Ray Conference,

30 July- 6 August, 2015

The Hague, The Netherlands

${ }^{*}$ Speaker. 
Born from the decays of particles created in the interaction of primary cosmic rays with air nuclei, atmospheric neutrinos are a continuous source of both neutrino and antineutrinos with energies ranging from $100 \mathrm{MeV}$ to more than $100 \mathrm{TeV}$. At the same time, since the arrival of these cosmic rays to the Earth is roughly isotropic, the resulting neutrino flux can be seen from all directions by terrestrial experiments. That is, experiments observing this flux sample neutrinos that have traveled from $\mathrm{O}(10) \mathrm{km}$ to more than $10,000 \mathrm{~km}$ before detection. This variety of both pathlengh, $L$, and energy, $E$, lead to the discovery of neutrino oscillations, which are known to be a function of $L / E$ to first order, using atmospheric neutrinos. Since that observation our knowledge of the PMNS mixing paradigm has improved greatly, with its three main mixing angles and two independent mass differences now measured and known to be non-zero.

Despite these successes there remain open questions in neutrino mixing, many of which can be addressed, at least in part, by atmospheric neutrinos. At the forefront is the question of whether the neutrino mass hierarchy is normal or inverted. Due to their interactions with the Earth's matter, atmospheric neutrinos are indeed sensitive to the sign of the mass hierarchy. Additionally, whether or not neutrino oscillations violate $\mathrm{CP}$ symmetry and whether or not the atmospheric mixing angle $\theta_{23}<45^{\circ}$ are two unresolved issues to which these neutrinos are sensitive.

Stepping outside of the PMNS framework, atmospheric neutrinos can shed light on more exotic mixing scenarios. While recent measurements from short-baseline beam experiments show hints at the existence of a fourth, "sterile," neutrino state [1], the effects of such a state can also be expected to appear as a sub-dominant contribution to the oscillated atmospheric neutrino flux. Further, as oscillations are an interferrometric effect they are also sensitive to possible Lorentzinvariance violating phenomena. Since such operators are expected to induce oscillations that are a function of just $L$ or of $L \times E$ atmospheric neutrinos are an ideal source to search for such effects. In what follows searches for evidence of both these and sterile neutrino oscillations as well as measurements in the standard oscillation framework by Super-Kamiokande are presented.

\section{Detector and Data Set}

Super-Kamiokande is a 50 kiloton water Cherenkov detector optically separated into an inner detector (ID), which serves as the primary target, and an outer detector (OD) used to veto external backgrounds. The detector itself is a $41.4 \mathrm{~m}$ diameter by $39.3 \mathrm{~m}$ tall right cylinder with a steel structure offset from the wall of this tank by $2 \mathrm{~m}$. This structure holds 11,146 inward-facing 20 inch photomultiplier tubes (PMTs), which view the ID, and 1,885 8 inch PMTs that view the OD. A $55 \mathrm{~cm}$ dead space separates the two PMT planes. Cherenkov radiation produced by charged particles traveling through the detector's water is collected by the PMTs and used to reconstruct physics events. More detailed information on the detector and its calibration are presented in [2].

Atmospheric neutrino data are separated into three topologically distinct categories, fully contained (FC), partially contained (PC), and upward-going muons (Up $\mu$ ). Fully contained interactions, which are events whose primary vertex is located within the ID's 22.5 kiloton fiducial volume, are divided into subsamples based on their number of reconstructed Cherenkov rings, their energy, the particle ID (PID) of their most energetic ring and the number of observed Michel electrons. PC events similarly have a fiducial vertex but have energy deposition in the OD consistent with a particle entering from the ID. Upward-going muon events are produced by neutrino interac- 
tions in the rock below Super-K which have enough energy to enter the detector. After all selections there are 19 analysis samples.

\section{PMNS Oscillations}
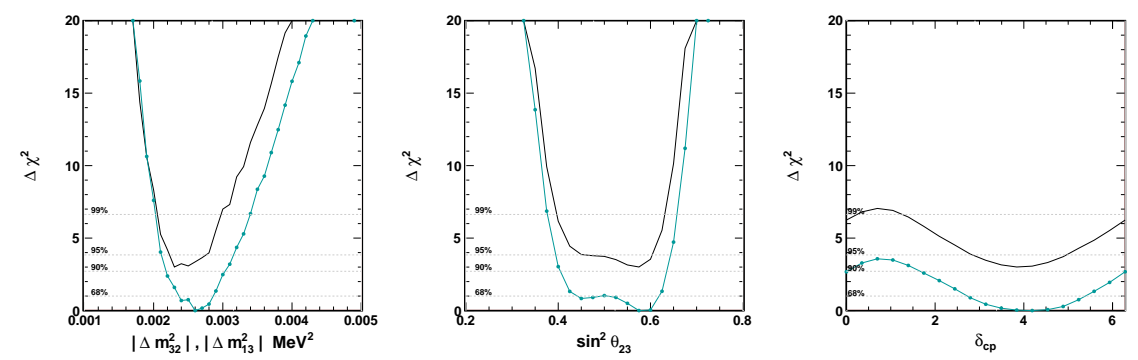

Figure 1: Parameter constraints from the Super-K Super-K atmospheric neutrino data. The inverted hierarchy fit result is shown in black lines, has been offset from the normal hierarchy result, shown in blue, by the difference in their minimum $\chi^{2}$ values.

\subsubsection{Standard Oscillations}

Atmospheric neutrino oscillations are dominated by the effects of the "atmospheric" mixing angle, $\theta_{23}$, and mass splitting $\Delta m_{32,13}^{2}$, which drive oscillations of $v_{\mu}$ into (primarily unseen) $v_{\tau}$ in the Super-K data [3]. Nonetheless oscillations with the third neutrino, $v_{e}$, are expected to play a role in the atmospheric neutrino data due to the effects of $\theta_{13}$ and the Earth's matter. Resonant enhancement of the $v_{\mu} \rightarrow v_{e}$ oscillation probability is expected at energies between $2-10 \mathrm{GeV}$ for upward-going neutrinos passing through the core and mantle. Importantly, this enhancement depends both on the sign of the mass hierarchy and the CP state of the neutrino: for a normal (inverted) mass hierarchy the resonance exists for neutrinos (antineutrinos) only. Since there are
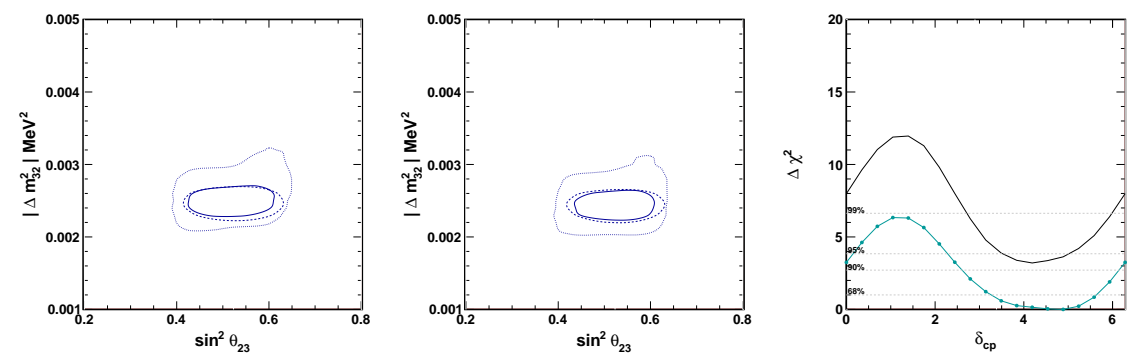

Figure 2: Parameter constraints from a combined fit of Super-K atmospheric neutrino data and a model of the T2K experiment. The left two figures show constraints using atmospheric neutrino data alone (dashed), the T2K model (dotted) alone, and their combination (solid). The normal (inverted) hierarchy result is shown in the first (second) plot from the left. Constraints on the $\delta_{C P}$ parameter from the combined fit with the inverted hierarchy fit (black) offset from the normal hierarchy result (blue, markers) by the difference in their minimum $\chi^{2}$ values appear in the right figure. 


\begin{tabular}{lcccc}
\hline \hline Fit & $\chi_{\min }^{2}$ & $\delta_{c p}$ & $\sin ^{2} \theta_{23}$ & $\Delta m_{32,13}^{2}\left[\mathrm{eV}^{2}\right]$ \\
\hline SK NH & 582.4 & 4.19 & 0.575 & $2.6 \times 10^{-3}$ \\
SK IH & 585.4 & 3.84 & 0.575 & $2.3 \times 10^{-3}$ \\
\hline SK+T2K NH & 651.5 & 4.89 & 0.525 & $2.5 \times 10^{-3}$ \\
SK+T2K IH & 654.7 & 4.19 & 0.550 & $2.4 \times 10^{-3}$ \\
\hline \hline
\end{tabular}

Table 1: Fit summary for the normal (NH) or inverted (IH) mass hierarchy analysis using either the Super-K atmospheric neutrino data only (517 d.o.f.), or combining it with the T2K model (585 d.o.f.).

both neutrinos and antineutrinos in the atmospheric flux the ability to separate the two provides enhanced hierarchy sensitivity. A description of the Super-K separation method may be found in [4].

In the Super-K atmospheric oscillation analysis all available data, including both $e$-like and $\mu$-like samples, are used in a simultaneous fit for $\theta_{23}, \Delta m_{23}^{2}$, and $\delta_{c p}$. Both hierarchy assumptions are tested. The value of $\sin ^{2} \theta_{13}$ has been fixed to 0.0238 based on [5] and its uncertainty has been included as a systematic error. Uncertainties in the flux and cross section models, as well as the detector's performance are included as 154 sources of systematic uncertainty in the fit. The data indicate a weak preference for the normal mass hierarchy, $\chi_{I H}^{2}-\chi_{N H}^{2}=3.0$. Table 1 summarizes the best fit information for both hierarchy tests and $\Delta \chi^{2}$ distributions for each measured parameter appear in Figure 1. It should be noted that the size of the resonance-enhanced oscillation probability depends also on the value of $\sin ^{2} \theta_{23}$ and is similarly modulated, though to a lesser degree, by the value of $\delta_{c p}$. If the former is 0.6 then the expected increase in the upward going multi-GeV event $e$-like rate is $17 \%$ compared to $8 \%$ if it is 0.4 . Super-K is able to constrain the atmospheric mixing parameters by using both its $e$-like and $\mu$-like data, but relative to fixed-beam experiments its sensitivity is limited. However, incorporating constraints from more precise measurements can be used to augment the hierarchy sensitivity of the Super-K analysis.

The atmospheric mixing parameters have been measured to a high degree of precision by the T2K [6] and MINOS [7] experiments. Since the T2K experiment uses Super-K as its far detector and the event simulation and reconstruction are common between beam and atmospheric neutrino events, it is straightforward to build a model of the T2K experiment using only information in the literature and atmospheric analysis tools. It is further possible to construct systematic error correlations between the atmospheric sample and a T2K model.

A model of the T2K experiment's $v_{e}$ appearance [8] and $v_{\mu}$ disappearance [6] analysis samples has been constructed using atmospheric neutrino tools. Publicly available beam flux information [9] is used to reweight the Super-K atmospheric neutrino MC to produce a beam MC. This MC is then passed through the same selection criteria used in T2K's official analyses. Data and systematics are taken from the literature [6, 8]. Independent fits of each model sample were performed to confirm they can successfully reproduce official results from the collaboration.

Both model samples are then fit together with the 19 Super-K analysis samples to directly incorporate T2K's constraints into the fit. Full correlations between relevant systematic error parameters, such as those in the cross section model, are included. The resulting contours are shown in Figure 2 and the best fit information is listed in Table 1. Including the T2K model strengthens 
Super-K's preference for the normal mass hierarchy slightly, $\chi_{I H}^{2}-\chi_{N H}^{2}=3.2$, but it remains insignificant. It is worth nothing that while both T2K and Super-K have a preference for $\delta_{c p} \sim 3 \pi / 2$, which is strengthened when they are fit together, CP-conservation is allowed at at least the $90 \%$ confidence level.

\section{Sterile Neutrino Oscillations}
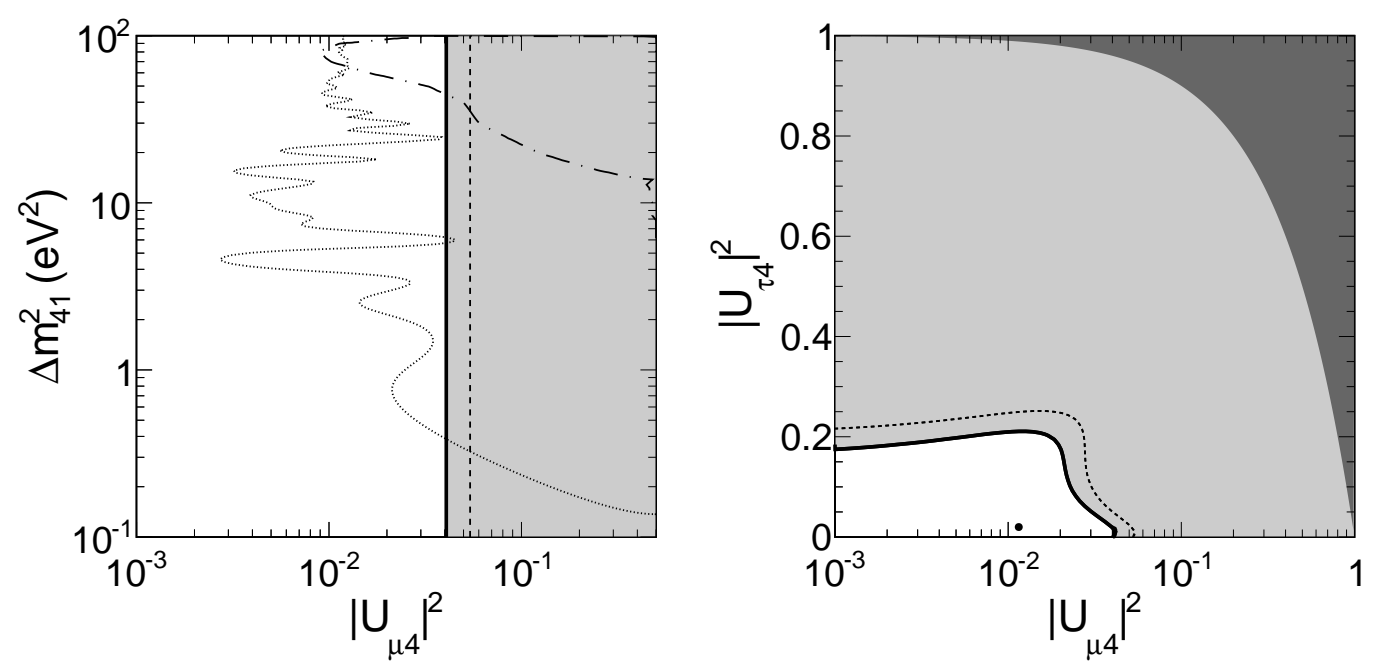

Figure 3: (Left) Upper imits on $\left|U_{\mu 4}\right|^{2}$ and on $\left|U_{\mu 4}\right|^{2}$ vs $\left|U_{\tau 4}\right|^{2}$ (right) from Super-K. The 90\% and 95\% C.L. limits are shown by the solid and dashed lines, respectively. The light gray region is excluded at $90 \%$ and the dark gray region is disallowed by unitarity [11].

Recently there have been a number of experimental hints to suggest the existence of an additional "sterile" neutrino mass state, and an accompanying third mass difference, $\Delta m_{s}^{2} \sim 1 \mathrm{eV}^{2}$ (c.f. [1] and its references). Despite having no weak interactions it is possible for such a state to influence the Super-K atmospheric neutrino data via oscillations between it and the standard neutrino flavors.

Atmospheric neutrinos are essentially insensitive to the exact number of sterile neutrinos. When $\Delta m_{s}^{2}>0.1 \mathrm{eV}^{2}$ the sterile oscillations are sufficiently fast that they are similarly not sensitive to the exact value of the mass splitting. However a non-zero mixing angle between the standard neutrinos and any sterile neutrinos is expected to produce spectral distortions in the atmospheric neutrino flux or to suppress the flux in some channels overall. Extensions of the PMNS oscillation framework to include sterile states expand the mixing matrix to include terms like $U_{e 4}, U_{\mu 4}$, and $U_{\tau 4}$ that describe mixing with standard neutrinos. Since sterile neutrinos do not participate in the weak interaction they lack NC interactions and, relative to the other neutrinos, they are subject to an additional effective potential, $V_{s}= \pm\left(G_{F} / \sqrt{2}\right) N_{n}$ when traveling through matter. In this expression $N_{n}$ is the local neutron density and $G_{F}$ the Fermi constant. Note there is no dependence on the proton or electron densities since the contributions of these to the NC cross section are equal and opposite for neutral matter. Here only one sterile state is assumed, without any $v_{e} \leftrightarrow v_{s}$ mixing, and oscillation probabilities are computed following [10]. 


\begin{tabular}{lcccc|c}
\hline \hline Fit & $\left|U_{\mu 4}\right|_{\text {best }}^{2}$ & $\left|U_{\tau 4}\right|_{\text {best }}^{2}$ & $\left|U_{\mu 4}\right|_{\text {lim }}^{2}$ & $\left|U_{\tau 4}\right|_{\text {lim }}^{2}$ & $\chi_{\min }^{2}$ (478 d.o.f.) \\
\hline No- $_{e}$ & 0.012 & 0.021 & - & $<0.18$ & 531.1 \\
Sterile $_{\text {Vacuum }}$ & 0.016 & - & $<0.041$ & - & 532.1 \\
\hline \hline
\end{tabular}

Table 2: Best fit information from the sterile oscillation analyses assuming no $v_{e}$ oscillations or no sterile

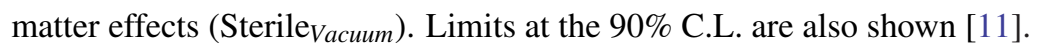

The search for sterile neutrino oscillations is done in two parts, each assuming a different approximation. Unfortunately it is not computationally feasible to unify these two domains. For the first analysis, $v_{e}$ oscillations are completely decoupled from the other active neutrinos. Specifically, $\theta_{13}=\theta_{23}=0$. Under this assumption atmospheric neutrinos are capable of simultaneously probing the effects of $\left|U_{\mu 4}\right|$, which is expected to decrease the overall $v_{\mu}$ survival probability, and $\left|U_{\tau 4}\right|$, which causes an energy dependent distortion of the $v_{\mu}$ flux through the sterile matter potential. For this reason the correlation of the Super-K $\mu$-like samples with the $e$-like samples through systematic uncertainties in the flux model ultimately leads to a bias towards lower values of $\left|U_{\mu 4}\right|$ due to the now missing $\theta_{13}$-induced events discussed above. Constraints on $\left|U_{\tau 4}\right|$ are possible via its effect on the PC and $\mathrm{Up} \mu$ samples, both of which are enriched in $v_{\mu}$ interactions. The second analysis assumes regular PMNS mixing with a sterile neutrino but eliminates the sterile matter effect by assuming the Earth contains no neutrons. In this approximation an unbiased measure of $\left|U_{\mu 4}\right|$ is possible.

Both analyses use 18 of the 19 Super-K analysis samples for a total of 480 degrees of freedom. In both oscillation tests no evidence for oscillations with $\Delta m_{s}^{2} \sim 1 \mathrm{eV}^{2}$ is seen. Similarly there is no indication of any spectral distortions from potential sterile matter effects. As a result Super-K has placed limits on the relevant mixing parameters, which are summarized in Table 2. Figure 3 shows their corresponding allowed regions.

\section{Lorentz-invariance Violating Oscillations}

The interferrometric nature of atmospheric neutrino oscillations also makes them sensitive to effects from Lorentz-invariance violating (LV) processes. Though there is no indication of any violations of this symmetry at present, the central role it plays both in the standard model of particle physics and in general relativity make it an important avenue of experimental research. Indeed, an observation of LV would provide access physics at the Planck scale.

Lorentz-invariance violating effects may manifest in atmospheric neutrinos as sidereal variations in their oscillations or as distortions in the oscillated spectra. To study the latter, Super-K has undergone a search using the standard model extension (SME) [12]. This model is an effective field theory which contains the standard model, general relativity, and all possible LV operators and is observer independent. Within the SME the neutrino Hamiltonian is modified by a LV component,

$$
H_{L V}=\left(\begin{array}{ccc}
0 & a_{e \mu}^{T} & a_{e \tau}^{T} \\
\left(a_{e \mu}^{T}\right)^{*} & 0 & a_{\mu \tau}^{T} \\
\left(a_{e \tau}^{T}\right)^{*} & \left(a_{\mu \tau}^{T}\right)^{*} & 0
\end{array}\right)-E\left(\begin{array}{ccc}
0 & c_{e \mu}^{T T} & c_{e \tau}^{T T} \\
\left(c_{e \mu}^{T T}\right)^{*} & 0 & c_{\mu \tau}^{T T} \\
\left(c_{e \tau}^{T T}\right)^{*} & \left(c_{\mu \tau}^{T T}\right)^{*} & 0
\end{array}\right)
$$



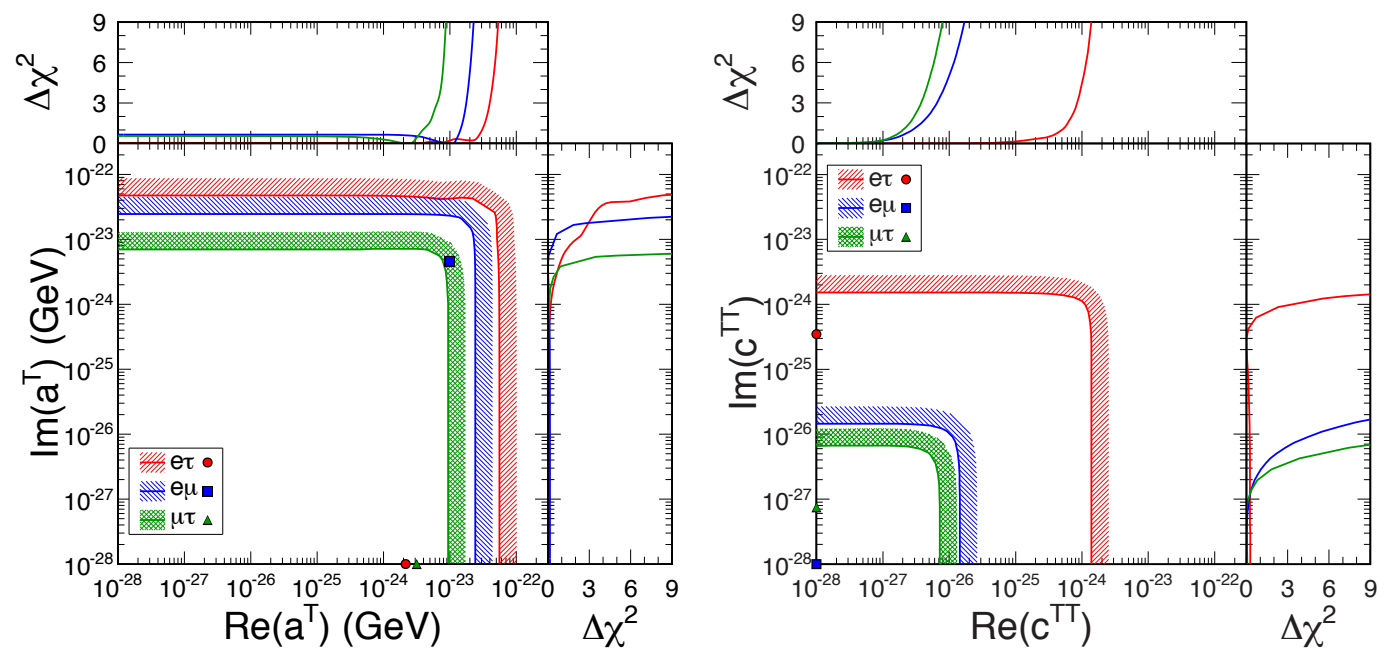

Figure 4: Allowed regions at the $95 \%$ confidence level for the Lorentz invariance violating parameters $a_{e \tau}^{T}$ (red), $a_{e \mu}^{T}$ (blue), $a_{\mu \tau}^{T}$ (green) are shown in the left figure. Similarly, those for $c_{e \tau}^{T T}$ (red), $c_{e \mu}^{T T}$ (blue), $c_{\mu \tau}^{T T}$ (green) appear in the right figure. Best fit points from the six fits appear as markes in the plots [14].

where $\mathrm{a}_{\alpha \beta}^{T}$ and $\mathrm{c}_{\alpha \beta}^{T T}$ are complex coefficients for isotropic LV operators. Broadly speaking, the $\mathrm{a}_{\alpha \beta}^{T}$ parameters produce oscillation effects proportional to the neutrino propagation distance, $L$, while the $\mathrm{c}_{\alpha \beta}^{T T}$ induce effects that depend on $L E$, where $E$ is the neutrino energy. For this reason, the large variety of pathlengths and energies found in atmospheric neutrinos make them particularly sensitive probes of these effects. Yet for the same reasons perturbative solutions to this Hamiltonian are invalid and the problem must be fully diagonalized. Here oscillation probabilities have been computed based on [13].

Event modest amounts of LV can have large effects on atmospheric neutrino oscillations, but generally they are expected to appear primarily in the multi-GeV (both $e$-like and $\mu$-like), PC, and Up $\mu$ samples. In the search for LV in the atmospheric neutrino data the standard oscillation parameters are held fixed at the best fit point of the standard PMNS analysis, with the exception of $\delta_{c p}$, which is allowed to vary freely in the fit. Both the real and imaginary components of each of the LV coefficients found in the Hamiltonian above are then fit for in the analysis. Note that only one coefficient is considered at a time, with the others held at zero. Unfortunately, the analysis finds no evidence for LV and as a result Super-K has placed limits three to seven orders of magnitude stronger than existing measurements. Additionally new limits on the $a_{\mu \tau}^{T}$ and $c_{\mu \tau}^{T T}$ parameters have been established.

\section{Conclusion}

Atmospheric neutrino data from Super-K have been used to study both standard PMNS and exotic oscillation scenarios. Constraints on the parameters of the former remain compelling and so for the data indicate a weak preference for the normal mass hierarchy. Though this measurement is statistically limited, an effort to boost the analysis sensitivity using constraints from a model of the T2K experiment have slightly strengthened the normal hierarchy preference. Searches for the 
existence of sterile or LV oscillations have yielded null results and stringent limits on the relevant parameters from both schemes have been set accordingly.

\section{References}

[1] A. A. Aguilar-Arevalo et al. [MiniBooNE Collaboration], Phys. Rev. Lett. 110, 161801 (2013) [arXiv:1207.4809 [hep-ex], arXiv:1303.2588 [hep-ex]].

[2] K. Abe et al. [Super-Kamiokande Collaboration], Nucl. Instrum. Meth. A 737, 253 (2014) [arXiv:1307.0162 [physics.ins-det]].

[3] K. Abe et al. [Super-Kamiokande Collaboration], Phys. Rev. Lett. 107, 241801 (2011) [arXiv:1109.1621 [hep-ex]].

[4] Ka Pik Lee, Study of the neutrino mass hierarchy with the atmospheric neutrino data observed in Super-Kamiokande, Ph.D. thesis, The University of Tokyo (2012).

[5] K. A. Olive et al. [Particle Data Group Collaboration], Chin. Phys. C 38, 090001 (2014).

[6] K. Abe et al. [T2K Collaboration], Phys. Rev. Lett. 111, no. 21, 211803 (2013) [arXiv:1308.0465 [hep-ex]].

[7] P. Adamson et al. [MINOS Collaboration], Phys. Rev. Lett. 112, 191801 (2014) [arXiv:1403.0867 [hep-ex]].

[8] K. Abe et al. [T2K Collaboration], Phys. Rev. Lett. 112, 061802 (2014) [arXiv:1311.4750 [hep-ex]].

[9] K. Abe et al. [T2K Collaboration], Phys. Rev. D 87, no. 1, 012001 (2013) [Phys. Rev. D 87, no. 1, 019902 (2013)] [arXiv:1211.0469 [hep-ex]].

[10] M. Maltoni and T. Schwetz, Phys. Rev. D 76, 093005 (2007) [arXiv:0705.0107 [hep-ph]].

[11] K. Abe et al. [Super-Kamiokande Collaboration], Phys. Rev. D 91, 052019 (2015) [arXiv:1410.2008 [hep-ex]].

[12] V. A. Kostelecky, Phys. Rev. D 69, 105009 (2004) [hep-th/0312310].

[13] J. S. Diaz (2014), IUHET 585.

[14] K. Abe et al. [Super-Kamiokande Collaboration], Phys. Rev. D 91, 052003 (2015) [arXiv:1410.4267 [hep-ex]]. 